REVISTA CIENCIAS BIOMÉ DICAS

ARTÍCULOS ORIGINALES

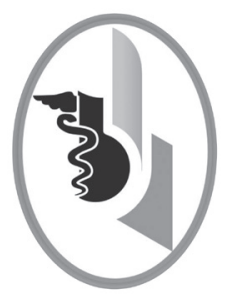

\title{
CARACTERIZACIÓN DE LOS RESULTADOS ADVERSOS MATERNOS Y NEONATALES LUEGO DE OPERACIÓN CESÁREA EN LA E.S.E. CLÍNICA DE MATERNIDAD RAFAEL CALVO
}

\author{
CHARACTERIZATION OF THE MATERNAL AND NEONATAL \\ ADVERSE OUTCOMES AFTER CAESAREAN SECTION IN THE \\ CLINICA DE MATERNIDAD RAFAEL CALVO
}

\author{
Rubio-Hoyos Sandra $\mathrm{M}^{1}$ \\ Méndez-Rodríguez Rogelio² \\ Contreras-Arrieta Sandra ${ }^{3}$
}

Correspondencia: sama-2570@hotmail.com

Recibido para evaluación: octubre - 20 - 2014. Aceptado para publicación: octubre - 15-2015.

\section{RESUMEN}

Introducción: la Organización Mundial de la Salud (OMS) recomienda que la incidencia de cesárea no supere el 15\%; sin embargo, su uso se ha incrementado en las dos últimas décadas, lo cual implica un problema de salud pública.

Objetivo: caracterizar los resultados adversos maternos y neonatales de la cesárea en la Clínica Maternidad Rafael Calvo (CMRC) de Cartagena Colombia.

Materiales y métodos: estudio descriptivo de corte transversal, donde se caracterizaron los resultados adversos maternos y neonatales posteriores a cesárea.

Resultados: se realizaron 1.804 cesáreas (46.5\%) y 2.073 partos vaginales $(53.5 \%)$. Las indicaciones más frecuentes para cesárea fueron cesárea anterior $(35.5 \%)$, desproporción céfalo pélvica (30.6\%) y oligoamnios (10.8\%). Los resultados adversos maternos más frecuentes fueron hemorragia que requirió transfusión $(9.17 \%)$, hematoma en la herida quirúrgica (3.28\%) e ingreso a UCI (1.5\%). Los resultados neonatales adversos más frecuentes fueron APGAR $<7$ al minuto $(21.3 \%)$, taquipnea transitoria del recién nacido (9.6\%) y APGAR<7 a los $5^{\prime}(4.3 \%)$. El control prenatal (CNP) fue factor protector para hemorragia uterina que requirió transfusión (OR crudo 0.54 , IC $0.38-0.76$, OR ajustado 0.53 IC $0.38-0.76$ ), hemorragia uterina que requirió histerectomía (OR crudo 0.20, IC $0.01-0.72$, OR ajustado 0.11 , IC $0.11-0.72$ ), shock obstétrico (OR crudo 0.06, IC $0.00-0.55$, OR ajustado, 0.04 , IC $0.04-0.38$ ) y destino (alojamiento conjunto) (OR ajustado 0.66 IC $0.49-0.90$ ), APGAR $<7$ a $1^{\prime}$ (OR ajustado 0.78 , IC, $0.44-0.72$ ), APGAR $<7$ a los $5^{\prime}$ (OR ajustado 0.48 , IC $0.28-0.82$ ) y la presencia de síndrome de distrés respiratorio (OR crudo 0.28, IC, $0.15-0.51$; OR ajustado 0.53 , IC $0.28-0.98$ ).

Conclusiones: la proporción de cesáreas que se realizan en la CMRC (46,5\%) es superior al 15\% recomendado por la OMS. La operación cesárea conlleva al aumento de morbilidad materno- fetal. EI CNP se comportó como factor protector frente a la aparición de resultados adversos maternos y neonatales. Rev.cienc.biomed. 2015;6(2):241250

\footnotetext{
Médico. Estudiante postgrado. Ginecología y Obstetricia. Facultad de Medicina. Universidad de Cartagena. Colombia.

Médico. Especialista en Ginecología y Obstetricia. Docente Departamento de Ginecología y Obstetricia. Universidad de Cartagena. Colombia.

Estudiante pregrado. Facultad de Medicina. Universidad de Cartagena. Colombia.
} 
PALABRAS CLAVE

Cesárea; Obstetricia ; Hemorragia.

\section{SUMMARY}

Introduction: the World Health Organization (WHO) recommends that the incidence of cesarean must not exceed $15 \%$; however, its use has increased in the last two decades, which implies a public health problem.

Objective: to characterize the maternal and neonatal adverse outcomes of caesarean in the Clínica Maternidad Rafael Calvo (CMRC),Cartagena-Colombia.

Methodology: a descriptive cross-sectional study, where after cesarean maternal and neonatal adverse outcomes were characterized.

Results: 1.804 cesarean sections (46.5\%) and 2.073 vaginal deliveries $(53.5 \%)$ were performed. The most common indications for cesarean section were before cesarean $(35.5 \%)$, cephalo pelvic disproportion $(30.6 \%)$ and oligoamnios $(10.8 \%)$. The most common adverse maternal outcomes were bleeding requiring transfusion $(9.17 \%)$, surgical wound hematoma (3.28\%) and admission to ICU $(1.5 \%)$. The most common adverse neonatal outcomes were APGAR $<7$ per minute $(21.3 \%)$, transient tachypnea of the newborn (9.6\%) and Apgar $<7$ at $5{ }^{\prime}(4.3 \%)$. Prenatal care (PNC) was protective factor for uterine bleeding requiring transfusion (OR crude $0.54, \mathrm{CI} 0.38$ to 0.76 , OR adjusted 0.53 IC 0.38 to 0.76 ), uterine bleeding which required hysterectomy (OR crude 0.20 , CI 0.01 to 0.72 , OR adjusted 0.11 , IC: 0.11 to 0.72 ), obstetric shock (OR crude 0.06 , IC: 0.00 to 0.55 ,OR adjusted 0.04 , CI 0.04 to 0.38 ) and destination (rooming) (OR 0.66, IC 0.49 to 0.90 ), APGAR $<7$ at 1 '( OR 0.78 IC 0.44 to 0.72 ), Apgar $<7$ at 5 '(adjusted OR 0.48 , IC 0.28 to 0.82 ) and the presence of respiratory distress (OR crude 0,28 IC, 0.15 to 0.51 ; OR adjusted 0.53 , IC 0.28 to 0.98 )

Conclusions: the proportion of cesarean sections performed in the CMRC $(46.5 \%)$ is above $15 \%$ recommended by WHO. Cesarean section leads to increased maternal and fetal morbidity. The CNP acted as a protective factor against the occurrence of adverse maternal and neonatal outcomes. Rev.cienc.biomed. 2015;6(2):241-250

\section{KEYWORDS}

Cesarean; Obstetric; Hemorrhage.

\section{INTRODUCCIÓN}

El dilema que más comúnmente tiene que enfrentar el obstetra en su práctica diaria es tener que decidir si realiza o no una operación cesárea. La misma tiene como objetivo la extracción del producto de la gestación, así como de la placenta y sus anexos, a través de una incisión en el hipogastrio que permite acceder al útero. La resolución quirúrgica de los eventos obstétricos a través de la operación cesárea constituye uno de los avances más importantes de la medicina perinatal contemporánea y ha tenido indiscutiblemente un impacto extraordinario en la disminución de la mortalidad materna y perinatal.

No se discute la validez de la operación cesárea cuando está de por medio una indicación absoluta. Sin embargo, en la época reciente ha ocurrido un aumento considerable en la práctica innecesaria de la operación cesárea $(1,2)$, que ciertamente no es inocua (3-7) y conlleva riesgos inherentes, quirúrgicos y anestésicos, con lo que esta técnica operatoria pierde su enorme efecto benéfico de salvar vidas de madres y recién nacidos cuando está bien indicada.

De hecho, la Organización Mundial de la Salud estima que la tasa ideal de cesáreas debe ser menor al $15 \%(8,9)$; sin embargo, no existe evidencia suficiente para hacer tal aseveración y algunos autores consideran que no es pertinente establecer una tasa de cesáreas ideal, ya que la misma es consecuencia de una decisión clínica basada en valores y condiciones individuales de la paciente (10). Al mismo tiempo, existe evidencia que la mortalidad perinatal disminuye en 1.61 por cada 1.000 nacimientos, por cada $1 \%$ de incremento de cesáreas.

Esta disminución se presenta con estas características en tasas de cesárea que van desde 0 hasta $8 \%$, tasas superiores a este porcentaje producen una disminución me- 
nos marcada de la mortalidad perinatal, de hecho, porcentajes superiores a $12 \%$ no reflejan disminución de la tasa de mortalidad intraparto (10). En Colombia, la tasa de cesáreas está entre $27 \%$ y 34\% (11) y en instituciones privadas llega hasta $67 \%$. Los mayores porcentajes de cesáreas del país se presentan principalmente en la región Caribe (12).

Es por ello que actualmente se busca revertir esta tendencia en alza, y se tiende a reducir los índices de incidencia de cesáreas tanto en el sector público como en el privado, realizándolas solo bajo indicaciones médicas indispensables, entendiéndose esto como una disminución en los costos hospitalarios, mejor atención del embarazo y trabajo de parto que conlleva a menores índices quirúrgicos con mayor beneficio materno y neonatal (13). El objetivo del estudio fue caracterizar los resultados adversos maternos y neonatales posteriores a la realización de cesárea.

\section{MATERIALES Y MÉTODOS}

Se realizó estudio descriptivo de corte transversal. La población de estudio consistió en la totalidad de pacientes a las que se les realizó una cesárea en el período comprendido entre el 1 de noviembre de 2014 hasta el 30 de abril de 2015 para un total de 1.804 pacientes. Se excluyeron aquellas pacientes con diagnóstico previo de óbito fetal (Gráfica No 1). Para el análisis estadístico se obtuvieron medidas de tendencia central para variables cuantitativas y para las variables cualitativas se obtuvieron frecuencias relativas y absolutas. Las variables categóricas se agruparon en variables dicotómicas para el análisis de causalidad a través de Odds Ratio utilizando la regresión logística.

\section{RESULTADOS}

Entre el período comprendido desde el 1 de noviembre de 2014 hasta el 30 de abril de 2015 se realizaron 1.804 cesáreas $(46.5 \%)$ y 2.073 partos vaginales (53.5\%). Del número de cesáreas mencionadas se excluyeron aquellas pacientes con diagnóstico de óbito fetal.

La edad promedio fue de $23.2 \pm 5.7$ años, con índice de masa corporal 24.96 44.07. El promedio de controles prenatales fue de $4.22 \pm 2.0$ controles. La edad gestacional promedio al momento de la cesárea fue de $38 \pm 2.23$ semanas. El tiempo operatorio fue de $44.59 \pm 9.52$ minutos, con promedio de evaluación del dolor postquirúrgico de $5.55 \pm 0.97$ (según escala análoga del dolor). La estancia hospitalaria materna promedio fue de $2.27 \pm 1.23$ días. El peso neonatal promedio fue de $3103.66 \pm 554.20$ gramos, mientras que la estancia hospitalaria del recién nacido fue de $1.58 \pm 1.19$ días.

Gráfica $\mathbf{N}^{\circ}$ 1. Flujograma de selección.

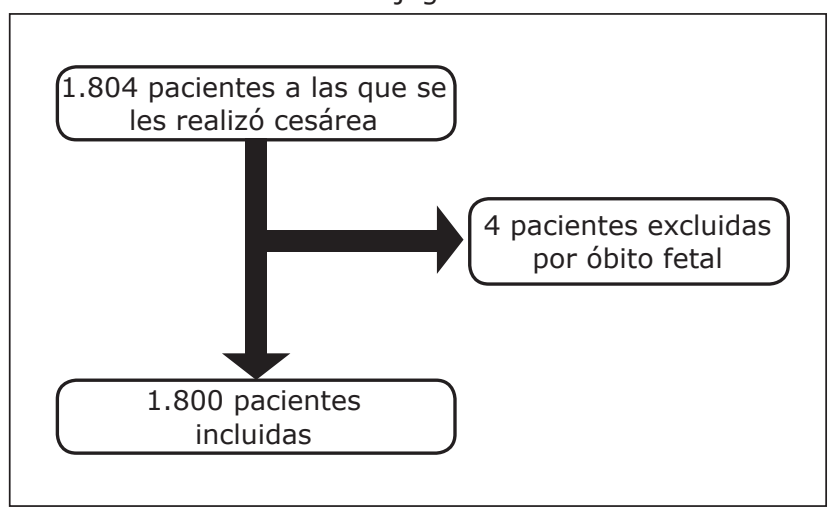

Gráfica N². Indicación para cesárea.

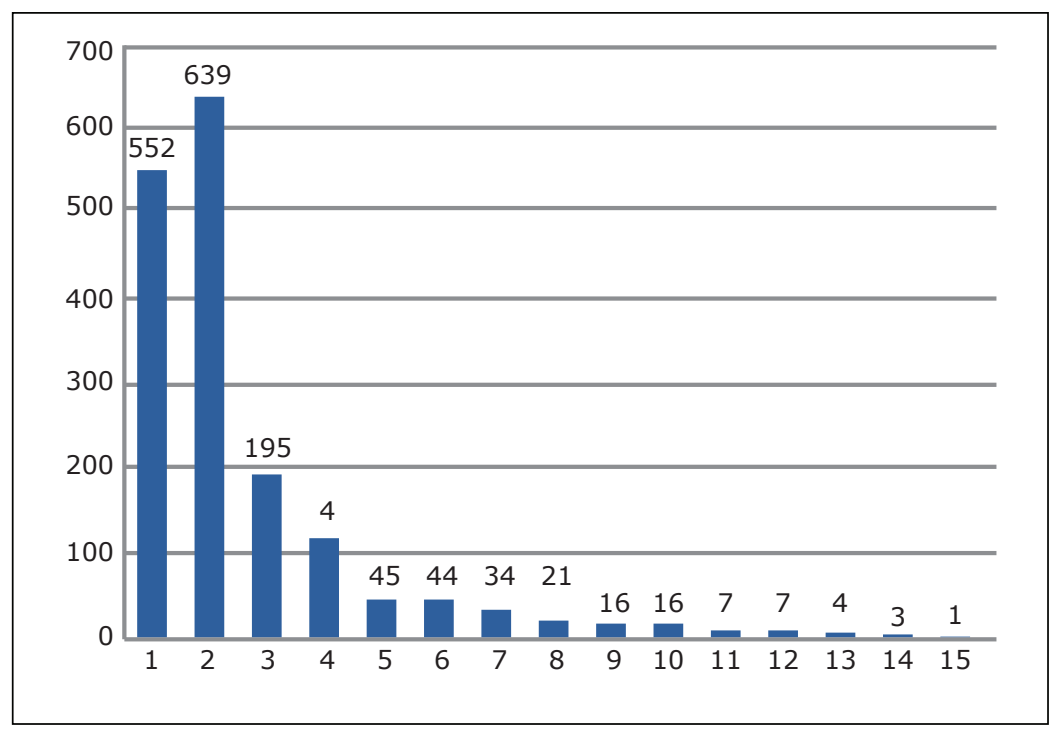

1. DCP, 2. Cesárea anterior, 3. Oligoamnios, 4. Trabajo de parto detenido, 5. Cuello desfavorable, 6. RCFU, 7. Enfermedad materna, 8. Expulsivo prolongado, 9. RPM, 10. DPPNI, 11. Cérvix distócico, 12. Placenta previa, 13. Malfonamación fela, 14. Pedido materno, 15. Sin diagnóstico. 
La Tabla N01 resume la frecuencia de las variables socioeconómicas y la Tabla No 2, los datos relacionados con la ficha ginecológica de las pacientes incluidas en el estudio. La distribución de las variables clínicas previas a la realización de la cesárea y las indicaciones de la misma está resumida en la Tabla No 3. La indicación más frecuente para cesárea fue cesárea anterior (35\%), seguida por desproporción céfalo-pélvica (30.6\%) y oligoamnios (10,8\%) (Gráfica No 2).

De las pacientes cuya indicación para cesárea fue oligoamnios, el $47 \%$ del total de pacientes con esta indicación tuvieron también el diagnóstico de ruptura prematura de membranas $(5.11 \%$ del total de las pacientes participantes en el estudio), mientras que en el $53 \%$ restante $(5.72 \%$ del total) los oligoamnios se presentaron de manera aislada o asociada a preeclampsia.

\begin{tabular}{|c|c|c|}
\hline \multicolumn{3}{|c|}{$\begin{array}{c}\text { TABLA No1. } \\
\text { DISTRIBUCIÓN PORCENTUAL DE LAS } \\
\text { CARACTERÍSTICAS SOCIODEMOGRÁFICAS } \\
\text { Y ANTECEDENTES GINECOBSTÉTRICOS } \\
\text { N=1800 }\end{array}$} \\
\hline Parámetro & $\mathbf{N}$ & $\%$ \\
\hline \multicolumn{3}{|c|}{$\begin{array}{l}\text { Estado civil } \\
\end{array}$} \\
\hline Casada & 202 & 11.2 \\
\hline Unión libre & 1491 & 82.8 \\
\hline Divorciada & 0 & 0 \\
\hline Viuda & 0 & 0 \\
\hline \multicolumn{3}{|c|}{ Zona de procedencia } \\
\hline Urbana & 1295 & 71.9 \\
\hline Rural & 505 & 28.0 \\
\hline \multicolumn{3}{|c|}{ Escolaridad } \\
\hline Primaria & 333 & 18.5 \\
\hline Secundaria & 1452 & 80.6 \\
\hline Universitaria & 15 & 0.8 \\
\hline \multicolumn{3}{|c|}{ Ocupación } \\
\hline Desempleada & 125 & 6.9 \\
\hline Empleada & 620 & 34.4 \\
\hline Ama de casa & 1055 & 58.5 \\
\hline \multicolumn{3}{|c|}{ Estrato socioeconómico } \\
\hline Bajo-bajo & 1 & 0.0 \\
\hline Bajo & 318 & 17.7 \\
\hline Medio Bajo & 1222 & 85.6 \\
\hline Medio & 258 & 99.9 \\
\hline Medio Alto & 1 & 0.0 \\
\hline \multicolumn{3}{|c|}{ Clasificación etário } \\
\hline Añosa & 103 & 5.7 \\
\hline Adolescente & 170 & 9.4 \\
\hline
\end{tabular}

\begin{tabular}{|c|c|c|}
\hline Ninguna & 1527 & 84.8 \\
\hline \multicolumn{3}{|c|}{ IMC } \\
\hline Bajo peso & 39 & 2.1 \\
\hline Normal & 822 & 45.7 \\
\hline Sobrepeso & 740 & 41.0 \\
\hline Obesidad & 199 & 11.0 \\
\hline \multicolumn{3}{|c|}{ Primigestante } \\
\hline $\mathrm{Si}$ & 801 & 44.5 \\
\hline No & 999 & 55.5 \\
\hline \multicolumn{3}{|c|}{ Gran multípara } \\
\hline $\mathrm{Si}$ & 22 & 1.2 \\
\hline No & 1788 & 98.7 \\
\hline \multicolumn{3}{|c|}{ Cesárea anterior } \\
\hline Si & 692 & 38.4 \\
\hline No & 1108 & 61.5 \\
\hline \multicolumn{3}{|c|}{ Motivo cesárea anterior } \\
\hline No sabe & 177 & 10.1 \\
\hline Indicación & 368 & 21.0 \\
\hline Sin indicación & 143 & 8.1 \\
\hline No aplica & 1102 & 61.2 \\
\hline \multicolumn{3}{|c|}{ Período intergenésico } \\
\hline$<18$ meses & 87 & 4.8 \\
\hline 18 meses - 5 años & 779 & 43.2 \\
\hline$>5$ años & 5 & 0.2 \\
\hline No aplica & 929 & 51.6 \\
\hline \multicolumn{3}{|c|}{ Control prenatal } \\
\hline Si & 1282 & 71.2 \\
\hline No/pobre control & 518 & 28.7 \\
\hline
\end{tabular}

\begin{tabular}{|c|c|c|}
\hline \multicolumn{3}{|c|}{$\begin{array}{c}\text { TABLA No } 2 . \\
\text { CARACTERÍSTICAS CLÍNICAS PREVIAS A } \\
\text { LA REALIZACIÓN DE LA CESÁREA } \\
\mathbf{N}=1800\end{array}$} \\
\hline Parámetro & $\mathrm{N}$ & $\%$ \\
\hline \multicolumn{3}{|c|}{ Placenta previa } \\
\hline No & 1788 & 99.3 \\
\hline Si & 12 & 0.6 \\
\hline \multicolumn{3}{|c|}{ Enfermedad materna previa } \\
\hline Ninguna & 1749 & 97.1 \\
\hline Si & 51 & 2.8 \\
\hline Otra & 31 & 1.7 \\
\hline Hipertensión arterial & 11 & 0.6 \\
\hline VIH/SIDA & 9 & 0.5 \\
\hline Diabetes & 1 & 0.0 \\
\hline Miomatosis uterina & 1 & 0.0 \\
\hline Anormalidad uterina & 0 & 0 \\
\hline \multicolumn{3}{|c|}{ Clasificación según edad gestacional } \\
\hline Término & 1544 & 85.7 \\
\hline No término & 256 & 14.2 \\
\hline Lejos del término & 8 & 0.4 \\
\hline Pretérmino & 220 & 12.2 \\
\hline
\end{tabular}




\begin{tabular}{|c|c|c|}
\hline Postérmino & 19 & 1.0 \\
\hline \multicolumn{3}{|c|}{ Embarazo múltiple } \\
\hline $\mathrm{Si}$ & 29 & 1.6 \\
\hline No & 1771 & 98.3 \\
\hline \multicolumn{3}{|c|}{ Inicio trabajo de parto } \\
\hline Espontáneo & 1264 & 70.2 \\
\hline Inducido & 93 & 5.1 \\
\hline Ninguno & 443 & 24.6 \\
\hline \multicolumn{3}{|c|}{ Tipo de motivación para cesárea } \\
\hline Urgencia & 1476 & 82.0 \\
\hline Programada & 324 & 18.0 \\
\hline \multicolumn{3}{|c|}{ Enfermedad asociada al embarazo } \\
\hline Ninguna & 989 & 54.9 \\
\hline Si & 811 & 45.0 \\
\hline RPM & 313 & 17.3 \\
\hline Preeclampsia & 296 & 16.4 \\
\hline Macrosomía fetal & 190 & 10.5 \\
\hline RCIU & 46 & 2.5 \\
\hline Infección intraamniótica & 24 & 1.3 \\
\hline Diabetes gestacional & 20 & 1.1 \\
\hline Malformación fetal & 3 & 0.1 \\
\hline Eclampsia & 3 & 0.1 \\
\hline \multicolumn{3}{|c|}{ Indicación para cesárea } \\
\hline DCP & 552 & 30.6 \\
\hline Cesárea anterior & 639 & 35.5 \\
\hline Oligoamnios & 195 & 10.8 \\
\hline Oligoamnios más RPM & 92 & 5.1 \\
\hline Oligoamnios sin RPM & 103 & 5.7 \\
\hline TP detenido & 119 & 6.6 \\
\hline Cuello desfavorable & 45 & 2.5 \\
\hline RCIU & 44 & 2.4 \\
\hline Enfermedad materna & 34 & 1.8 \\
\hline Expulsivo prolongado & 21 & 1.1 \\
\hline RPM & 16 & 0.8 \\
\hline DPPNI & 16 & 0.8 \\
\hline Cérvix distócico & 7 & 0.3 \\
\hline Placenta previa & 7 & 0.3 \\
\hline Malformación fetal & 4 & 0.2 \\
\hline Pedido materno & 3 & 0.1 \\
\hline Sin diagnóstico & 1 & 0.0 \\
\hline Cordón procúbito & 0 & 0 \\
\hline
\end{tabular}

\begin{tabular}{|l|c|c|}
\hline \multicolumn{3}{|c|}{ TABLA No 3.} \\
\multicolumn{2}{|c|}{$\begin{array}{c}\text { ADESULTADOS MATERNOS Y NEONATALES } \\
\text { ADVES }\end{array}$} \\
\hline $\begin{array}{l}\text { Resultados maternos } \\
\text { adversos }\end{array}$ & $\mathbf{N}$ & $\%$ \\
\hline $\begin{array}{l}\text { Hemorragia uterina que } \\
\text { requiere trasfusión }\end{array}$ & 165 & 9.1 \\
\hline $\begin{array}{l}\text { Hematoma en herida } \\
\text { obstétrica }\end{array}$ & 59 & 3.2 \\
\hline
\end{tabular}

\begin{tabular}{|c|c|c|}
\hline Ingreso a UCI & 27 & 1.5 \\
\hline Reintervención quirúrgica & 19 & 1.0 \\
\hline Reingreso & 16 & 0.8 \\
\hline $\begin{array}{l}\text { Dehiscencia de la herida } \\
\text { quirúrgica }\end{array}$ & 11 & 0.6 \\
\hline Shock séptico puerperal & 8 & 0.4 \\
\hline Sepsis puerperal & 8 & 0.4 \\
\hline Shock obstétrico & 7 & 0.3 \\
\hline $\begin{array}{l}\text { Hemorragia uterina que } \\
\text { requiere histerectomía }\end{array}$ & 6 & 0.3 \\
\hline Endometritis & 4 & 0.2 \\
\hline Lesión de órganos & 2 & 0.1 \\
\hline Vejiga & 2 & 100 \\
\hline Muerte intrahospitalaria & 1 & 0.6 \\
\hline Ruptura uterina & 1 & 0.6 \\
\hline Falla renal aguda & 1 & 0.6 \\
\hline Paro cardíaco & 1 & 0.6 \\
\hline Tratamiento antibiótico & 1800 & 100 \\
\hline Profilaxis antitrombótica & 33 & 1.8 \\
\hline Bajo peso al nacer & 142 & 7.8 \\
\hline \multicolumn{3}{|c|}{ Sexo } \\
\hline Femenino & 911 & 50.6 \\
\hline Masculino & 889 & 49.3 \\
\hline \multicolumn{3}{|c|}{ Destino del neonato } \\
\hline Alojamiento conjunto & 1543 & 85.7 \\
\hline $\begin{array}{l}\text { Unidad de Cuidados } \\
\text { Intensivos }\end{array}$ & 140 & 7.7 \\
\hline Cuidados básicos & 117 & 6.5 \\
\hline APGAR $<7$ al min & 384 & 21.3 \\
\hline Taquipnea transitoria & 173 & 9.6 \\
\hline APGAR $<7$ a los $5 \mathrm{~min}$ & 79 & 4.3 \\
\hline SDR & 54 & 3 \\
\hline Sepsis neonatal & 44 & 2.4 \\
\hline Hipoglicemia & 22 & 1.2 \\
\hline Muerte neonatal & 12 & 0.6 \\
\hline Laceraciones & 3 & 0.1 \\
\hline Ictericia neonatal & 4 & 0.2 \\
\hline SALAM & 4 & 0.2 \\
\hline
\end{tabular}

Los resultados adversos maternos y neonatales se resumen en la Tabla No 3. El resultado adverso materno más frecuente en el estudio fue la hemorragia que requirió trasfusión $(9.17 \%)$, seguido de hematoma en la herida quirúrgica $(3.28 \%)$ e ingreso a la UCI $(1.5 \%)$.

En cuanto a los resultados neonatales el $50.61 \%$ de los pacientes fue de sexo femenino y el $49.3 \%$ restante fue de sexo masculino. El $85.7 \%$ de los pacientes fueron destinados a alojamiento conjunto, el $7.7 \%$ a la unidad de cuidados intensivos neonatales 
y el $6.5 \%$ a cuidados básicos. El resultado neonatal adverso más frecuente fue APGAR $<7$ al minuto (21.3\%), taquipnea transitoria del recién nacido $(9.6 \%)$ y APGAR $<7$ a los $5 \min (4.3 \%)$.

Al buscar relación de asociación entre variables clínicas y los resultados adversos maternos y neonatales (Tabla 4), el control prenatal se comportó como factor protector de hemorragia uterina que requirió trasfusión (OR crudo 0.54, IC $0.38-0.76$, OR ajustado 0.53 IC $0.38-0.76$ ), hemorragia uterina que requirió histerectomía ( OR crudo 0.20, IC $0.01-0.72$, OR ajustado 0.11 , IC 0.11 0.72 ), shock obstétrico (OR crudo 0.06 , IC $0.00-0.55$, OR ajustado, 0.04, IC $0.04-$ 0.38 ) y frente al ingreso a UCI ( OR crudo 0.42, IC 0.18-0.99) con el OR crudo, pero no estadísticamente significativo al realizar la regresión logística.

En los resultados neonatales, el control prenatal fue factor protector para destino (alojamiento conjunto) (OR crudo 0.47, IC, 0.36 - 0.63; OR ajustado 0.66, IC $0.49-0.90)$, el APGAR <7 min (OR crudo 0.48 , IC 0.38 - 0.62; OR ajustado 0.78, IC, $0.44-0.72$ ), APGAR <7 a los 5 min (OR crudo 0.35, IC
$0.21-0.58$; OR ajustado 0.48 , IC 0.28 0.82 ) y la presencia de síndrome de distrés respiratorio (OR crudo 0.28 , IC, $0.15-0.51$; OR ajustado 0.53 , IC $0.28-0.98$ ). Adicionalmente, la cesárea programada se comportó como factor protector para el destino (OR crudo, IC $0.13-0.42$; OR ajustado 0.47 , IC $0.26-0.83)$, y resultó significativo en el OR crudo pero no en la regresión logística para APGAR $<7$ a los $5 \mathrm{~min}$ (OR crudo 0.62, IC, $0.44-0.86$, el APGAR < 7) a los $5 \mathrm{~min}$ (OR crudo 0.39 , IC $0.13-0.92$ ) y la TTRN (OR crudo 0.40 , IC $0.21-0.70)$. No hubo ninguna otra asociación significativa.

\section{DISCUSIÓN}

La Organización Mundial de la Salud establece que en ninguna región del mundo se justifica una incidencia de cesárea mayor del 10-15\%; no obstante, se ha incrementado su frecuencia en las dos últimas décadas (14). En nuestro país y en Latinoamérica la realidad dista mucho de esa recomendación, América es la región del mundo con la mayor tasa de cesáreas (31.9\% en el año 2011).

En Colombia, se encuentra una clara tendencia al incremento de la cesárea desde

\begin{tabular}{|c|c|c|c|c|}
\hline \multicolumn{5}{|c|}{$\begin{array}{l}\text { TABLA No } 4 . \\
\text { ASOCIACIÓN RESULTADOS ADVERSOS Y CONDICIONES CLÍNICAS }\end{array}$} \\
\hline \multicolumn{5}{|c|}{ HEMORRAGIA UTERINA QUE REQUIERE TRASFUSIÓN } \\
\hline & OR Crudo & IC $95 \%$ & OR Ajustado & IC $95 \%$ \\
\hline Control prenatal & 0.54 & $0.38-0.76$ & 0.53 & $0.38-0.76$ \\
\hline \multicolumn{5}{|c|}{ INGRESO A UCI } \\
\hline Control prenatal & 0.42 & $0.18-0.99$ & 0.13 & $0.29-1.48$ \\
\hline \multicolumn{5}{|c|}{ HEMORRAGIA UTERINA QUE REQUIERE HISTERECTOMÍA } \\
\hline Control prenatal & 0.20 & $0.01-1.40$ & 0.11 & $0.01-0.72$ \\
\hline \multicolumn{5}{|c|}{ SHOCK OBSTÉTRICO } \\
\hline Control prenatal & 0.06 & $0.00-0.55$ & 0.04 & $0.04-0.38$ \\
\hline \multicolumn{5}{|c|}{ DESTINO } \\
\hline Control prenatal & 0.47 & $0.36-0.63$ & 0.66 & $0.49-0.90$ \\
\hline \multicolumn{5}{|c|}{ APGAR $<7$ AL MINUTO } \\
\hline Control prenatal & 0.48 & $0.38-0.62$ & 0.56 & $0.44-0.72$ \\
\hline Cesárea anterior & 0.71 & $0.55-0.90$ & 0.78 & $0.60-1.01$ \\
\hline \multicolumn{5}{|c|}{ APGAR $<7$ A LOS 5 MINUTOS } \\
\hline Control prenatal & 0.35 & $0.21-0.58$ & 0.48 & $0.28-0.82$ \\
\hline Tipo cesárea & 0.39 & $0.13-0.92$ & 1.36 & $0.53-3.46$ \\
\hline \multicolumn{5}{|c|}{ SÍNDROME DE DISTRÉS RESPIRATORIO } \\
\hline Control prenatal & 0.28 & $0.15-0.51$ & 0.53 & $0.28-0.98$ \\
\hline \multicolumn{5}{|c|}{ TAQUIPNEA TRANSITORIA DEL RECIÉN NACIDO } \\
\hline Tipo cesárea & 0.40 & $0.21-0.70$ & 0.59 & $0.33-1.05$ \\
\hline
\end{tabular}


$24.9 \%$ en 1998 hasta $45.7 \%$ en el año 2013 (15). Al realizar un análisis por departamentos se encuentran diferencias acentuadas en las tasas de realización de cesárea en las diferentes regiones del país, siendo particularmente elevadas en la región $\mathrm{Ca}$ ribe colombiana, más exactamente en los departamentos de San Andrés, Atlántico, Bolívar y adicionalmente, los santanderes, aunque en todas las zonas del país se encuentran tasas por encima de la recomendación de la OMS.

En el estudio actual, la proporción de cesáreas realizadas en el período estudiado $(46.5 \%)$ supera ampliamente la recomendación de la OMS, incluso es ligeramente superior a los porcentajes reportados en el resto del país. Esto puede deberse al hecho que la CRMC es una institución de referencia en la región, motivo por el cual la tasa de cesáreas puede elevarse en virtud de las pacientes complicadas referidas de otras regiones cercanas.

El $44.5 \%$ de las pacientes estudiadas eran primigestantes al momento de la realización de la cesárea, y el $61.6 \%$ de la población no tenía antecedente de cesárea. En cuanto al índice de masa corporal, las pacientes clasificadas dentro de la categoría de sobrepeso y obesidad suman un 52\% (41\% y $11 \%$ respectivamente). Kominiarek et al. encontraron en su estudio que las tasas de cesárea aumentaron con todas las edades gestacionales en mujeres nulíparas, con una tasa de $11.1 \%$ en mujeres con IMC menor a 25 , comparadas con $33 \%$ cuando el IMC se encontraba entre 35 y 39.9 (16). Cada incremento de $1 \mathrm{~kg} / \mathrm{m}^{2}$ en el IMC aumentó el riesgo para la realización de una cesárea en mujeres nulíparas.

En este estudio, la indicación más frecuente para cesárea fue cesárea anterior (35.5\%), seguida por desproporción céfalo-pélvica $(30.6 \%)$ y oligoamnios $(10.8 \%)$. En el estudio realizado por Barriga-Moreno et al., el motivo más frecuente para la realización de una nueva cesárea es el antecedente de otra, seguido por estado fetal no satisfactorio, trastornos hipertensivos del embarazo, detención secundaria de la dilatación y el descenso (17).
El diagnóstico de cesárea previa correspondió al $26.4 \%$ de las cesáreas en el instituto materno infantil, seguidas en orden de frecuencia por estado fetal no satisfactorio con $15.5 \%$, trastornos hipertensivos del embarazo $15.5 \%$, detención secundaria de la dilatación $5.5 \%$ y ruptura prematura de membranas $4.7 \%$ (17). Barber et al. (18) analizaron las indicaciones de 10.757 mujeres a las que se le realizó operación cesárea entre 2003 y 2009 en un hospital universitario. Ellos encontraron que el estado fetal no satisfactorio durante el trabajo de parto (32\%) y la detención del trabajo de parto (18\%) contribuyeron en $50 \%$ al aumento en la tasa de primera cesárea.

De manera similar, Main et al., analizaron las tasas e indicaciones para cesárea en California-EE.UU. Estos autores se fijaron específicamente en mujeres con gestaciones únicas a término en presentación de vértice, donde la tasa de cesáreas fue de $28.1 \%$ en total con variaciones regionales que iban desde $21.5 \%$ a 33.5 y variaciones por hospital que iban del $10 \%$ al $50 \%$. La intolerancia fetal al trabajo de parto contribuyó con el 40-50\% de todas las cesáreas realizadas (19).

Como se mencionó anteriormente, la cesárea anterior fue el motivo más frecuente para la realización de una cesárea en el período de estudio. En la literatura médica está ampliamente aceptado que la cesárea anterior no es indicación, se considera que el parto vaginal en paciente con cesárea anterior es una alternativa segura, que no implica incremento en la morbimortalidad materno fetal. El riesgo de ruptura uterina es la principal preocupación, aunque esta situación no sobrepase el $1 \%$ de los casos (7-12). Lo estudios reportan que hasta un $82 \%$ de las pacientes con antecedente de cesárea podrían tener un parto vaginal (822 ), de hecho, la frecuencia de parto vaginal posterior a cesárea fue de $20 \%$ en un hospital de Colombia (20).

Sin embargo, existen unos requisitos que se deben cumplir para que el parto vaginal por cesárea sea posible, entre ellos tenemos un adecuado control prenatal, período intergenésico mayor de 18 meses, solo una cesárea anterior, incisión uterina transversa en la ce- 
sárea anterior, que la pelvis sea adecuada para el producto actual, que no existan cicatrices uterinas por cirugías ginecológicas previas, que el inicio del trabajo de parto sea espontáneo, vigilancia materno fetal durante el trabajo de parto, disponibilidad de equipo multidisciplinario conformado por ginecobstetra, anestesia, enfermería, banco de sangre y quirófano para la realización de una cesárea de emergencia, y adicionalmente, el consentimiento informado de la madre (21).

El oligoamnios fue la tercera causa más frecuente de cesárea, y la ruptura prematura de membranas fue causa del oligoamnios en el $47 \%$ ( $5 \%$ del total de la población) lo cual implica que la real indicación para la realización de la cesárea fue la ruptura prematura de membranas. Respecto a lo anterior, comúnmente se relaciona el oligoamnios con resultados fetales adversos, de hecho, en un metaanálisis de 18 estudios reporta una incidencia en aumento de cesárea por compromiso del bienestar fetal y puntajes bajos de Apgar a los 5 minutos en aquellas pacientes que cursaron con oligoamnios (22).

Puede ser que los resultados adversos estén relacionados con condiciones que afectan el embarazo y no el oligoamnios en sí mismo. Si bien algunos estudios sugieren que incluso en embarazos de alto riesgo el volumen de líquido amniótico no es un buen predictor de eventos adversos $(23,24)$.

En el presente estudio se encontró que el resultado adverso materno más frecuente fue la hemorragia que requirió trasfusión $(9,17 \%)$, seguido de hematoma en la herida quirúrgica (3.28\%) e ingreso a la UCI (1.5\%). Por otro lado, Villar et al. encontró un riesgo significativamente mayor de hemorragia obstétrica $(\mathrm{OR}=1.39$; IC 95\%: 1.10-1.76), histerectomía periparto $(\mathrm{OR}=4.73$; IC 95\%: 2.79-8.02), admisión a cuidados intensivos $(\mathrm{OR}=2.22$; IC 95\%: 1.45-3.40) y la necesidad de antibióticos periparto $(\mathrm{OR}=5.53$; IC 95\%: 3.77-8.10) en mujeres sometidas a cesárea comparadas con el parto vaginal, y concluyen que aunque la cesárea es efectiva al momento de reducir el riesgo de complicaciones en partos en podálica, cuando se trata de partos en cefálica aumenta la morbilidad severa materna y neonatal (25).
En el presente estudio se encontró que el control prenatal es factor protector frente a la aparición de hemorragia uterina que requiere trasfusión, ingreso a la UCI, hemorragia que requirió histerectomía y shock obstétrico. En la literatura actual se relaciona el adecuado control prenatal con la disminución de la morbimortalidad materno-fetal; un estudio latinoamericano concluyó que el control prenatal reduce intervenciones innecesarias durante el embarazo y el parto (26).

El riesgo de tratamiento antibiótico después del parto para mujeres a las que se le realizó cualquier tipo de cesárea fue cinco veces mayor que el de las mujeres con parto natural (25). En este estudio la totalidad de las pacientes a las que se les practicó la cesárea se les realizó, también, profilaxis antibiótica, lo cual sigue las recomendaciones internacionales (27).

Los resultados adversos más frecuentes en los neonatos nacidos por cesárea fueron el APGAR <7 minuto, taquipnea transitoria del recién nacido y $A P G A R<7$ a los 5 minutos, todas variables relacionadas con la morbilidad respiratoria en el recién nacido. CerianiCernadas et al. encontraron que la morbilidad respiratoria se asoció en forma independiente al nacimiento por cesárea (OR 1.75; IC $95 \%, 1.12-2.75$ ) y al sexo masculino (OR 1.75; IC95\% 1.10-2.78) (28).

En este estudio, el adecuado control prenatal se comportó como un factor protector frente a la hospitalización de los neonatos (unidad de cuidados intensivos y cuidados básicos), el APGAR $<7$ minuto, APGAR $<7$ a los 5 minutos y el síndrome de distrés respiratorio. Por otro lado, la cesárea programada (o electiva) se comportó como factor protector frente a la hospitalización del recién nacido, el APGAR $<7$ al minuto, APGAR $<7$ a los 5 minutos y la taquipnea transitoria del recién nacido, mientras que en el estudio conducido por Tita et al. se concluyó que la cesárea electiva antes de las 39 semanas de gestación es común y está asociada con morbilidad respiratoria y otros eventos adversos neonatales (29).

Un estudio más temprano mostró que la cesárea electiva antes de las 40 semanas de gestación estaba asociada con un aumento en las unidades de cuidados especial neo- 
natal por distrés respiratorio (mayormente por taquipnea transitoria del recién nacido) (30). Adicionalmente, a término, la incidencia de distrés respiratorio en niños nacidos por cesárea electiva fue mucho mayor que en aquellos nacidos por parto vaginal (31).

\section{CONCLUSIÓN}

La proporción de cesáreas que se realiza en la Clínica de Maternidad Rafael Calvo (46.5\%) es muy superior al $15 \%$ recomendado por la OMS, debido a la cesárea anterior, principalmente, por la renuencia por parte de los obstetras y las pacientes para intentar parto vaginal después de cesárea.

Se debe replantear la indicación de oligoamnios posterior a una RPM, para la realización de cesárea. El control prenatal es el factor protector más fuerte frente a la presentación de resultados adversos materno y neonatal.

La operación cesárea conlleva al aumento de morbilidad materno-fetal. Se necesitan estrategias multidisciplinarias que implican la vigilancia adecuada de las gestantes con y sin cesárea previa, en trabajo de parto espontáneo o inducido, con el fin de reducir la tasa de cesárea en la Clínica de Maternidad Rafael Calvo.

CONFLICTOS DE INTERESES: ninguno que declarar.

FINANCIACIÓN: recursos propios de los autores

\section{REFERENCIAS BIBLIOGRÁFICAS}

1. Villar J, Valladares E, Wojdyla D, Zavaleta N, Carroli G, Velazco A, et al. Caesarean delivery rates and pregnancy outcomes: the 2005 WHO global survey on maternal and perinatal health in Latin America. Lancet. 2006;367(9525):1819-29.

2. Souza JP, Gulmezoglu A, Lumbiganon P, Laopaiboon M, Carroli G, Fawole B, et al. Caesarean section without medical indications is associated with an increased risk of adverse short-term maternal outcomes: the 2004-2008 WHO Global Survey on Maternal and Perinatal Health. BMC Med. 2010;8:71.

3. Burrows LJ, Meyn LA, Weber AM. Maternal morbidity associated with vaginal versus cesarean delivery. Obstet Gynecol. 2004;103(5 Pt 1):907-12.

4. Chongsuvivatwong V, Bachtiar H, Chowdhury ME, Fernando S, Suwanrath C, Kor-Anantakul $O$, et al. Maternal and fetal mortality and complications associated with cesarean section deliveries in teaching hospitals in Asia. J Obstet Gynaecol Res. 2010;36(1):45-51.

5. Kamilya G, Seal SL, Mukherji J, Bhattacharyya SK, Hazra A. Maternal mortality and cesarean delivery: an analytical observational study. J Obstet Gynaecol Res. 2010;36(2):24853.

6. Liu S, Liston RM, Joseph KS, Heaman M, Sauve R, Kramer MS, et al. Maternal mortality and severe morbidity associated with low-risk planned cesarean delivery versus planned vaginal delivery at term. Canadian Medical Association Journal. 2007;176(4):455-60.

7. Deneux-Tharaux C, Carmona E, Bouvier-Colle MH, Breart G. Postpartum maternal mortality and cesarean delivery. Obstet Gynecol. 2006;108(3 Pt 1):541-8.

8. Sakala C. Medically unnecessary cesarean section births: introduction to a symposium. Soc Sci Med. 1993;37(10):1177-98.

9. Niino $Y$. The increasing cesarean rate globally and what we can do about it. Biosci Trends. $2011 ; 5(4): 139-50$.

10. Cyr RM. Myth of the ideal cesarean section rate: commentary and historic perspective. Am J Obstet Gynecol. 2006;194(4):932-6.

11. Profamila. Encuesta Nacional de Demografía y Salud ENDS 20102010 [cited 2015 Feb 1]. Available from:http://www.profamilia.org.co/encuestas/Profamilia/Profamilia/index. php?option=com_content\&view $=$ article\&id $=62 \&$ Itemid $=9$.

12.A. G. Partos atendidos por cesárea: Análisis de los datos de las Encuestas Nacionales de Demografía y Salud en Colombia 1995-2005. EAN. 2009;67:59-74.

13. Brown HC, Paranjothy S, Dowswell T, Thomas J. Package of care for active management in labour for reducing caesarean section rates in low-risk women. Cochrane Database Syst Rev. 2008(4):CD004907.

14. Clark SL, Belfort MA, Dildy GA, Herbst MA, Meyers JA, Hankins GD. Maternal death in the 21st century: causes, prevention, and relationship to cesarean delivery. Am J Obstet Gynecol. 2008;199(1):36 e1-5; discussion 91-2 e7-11.

15. (DANE) RdCDaNdE. Nacimientos por tipo de parto 2013 [cited 2015 Feb 1]. Available from: http://www.dane.gov.co/. 
16. Kominiarek MA, Vanveldhuisen $\mathrm{P}$, Hibbard J, Landy $\mathrm{H}$, Haberman $\mathrm{S}$, Learman $\mathrm{L}$, et al. The maternal body mass index: a strong association with delivery route. Am J Obstet Gynecol. 2010;203(3):264 e1-7.

17. Barriga-Moreno A B-SJ, Navarro-Vargas J. Indicación de Ccesárea en el Instituto Materno Infantil (IMI) Bogotá - Colombia. Serie de Casos Rev Fac Med. 2012;60(2):111-5.

18. Barber $\mathrm{EL}$, Lundsberg LS, Belanger K, Pettker CM, Funai EF, Illuzzi JL. Indications contributing to the increasing cesarean delivery rate. Obstet Gynecol. 2011;118(1):29-38.

19. King TL. Preventing primary cesarean sections: intrapartum care. Semin Perinatol. 2012;36(5):357-64.

20. Muñoz-Enciso J R-AE, Domínguez-Ponce G. Operación cesárea: cindicación justificante o preocupación justificada? Ginecol Obstet Mex. 2011;79(2):67-74.

21. Scott JR. Intrapartum management of trial of labour after caesarean delivery: evidence and experience. BJOG. 2014;121(2):157-62.

22. Chauhan SP, Sanderson M, Hendrix NW, Magann EF, Devoe LD. Perinatal outcome and amniotic fluid index in the antepartum and intrapartum periods: A meta-analysis. Am J Obstet Gynecol. 1999;181(6):1473-8.

23. Magann EF, Kinsella MJ, Chauhan SP, McNamara MF, Gehring BW, Morrison JC. Does an amniotic fluid index of $</=5 \mathrm{~cm}$ necessitate delivery in high-risk pregnancies? A case-control study. Am J Obstet Gynecol. 1999;180(6 Pt 1):1354-9.

24. Ott WJ. Reevaluation of the relationship between amniotic fluid volume and perinatal outcome. Am J Obstet Gynecol. 2005;192(6):1803-9; discussion 9.

25. Villar J, Carroli G, Zavaleta N, Donner A, Wojdyla D, Faundes A, et al. Maternal and neonatal individual risks and benefits associated with caesarean delivery: ulticentre prospective study. BMJ. 2007;335(7628):1025.

26. Soto C TH, Cabrera C, Marín M, Cabrera J, Da Costa M, Araneda H. Educación prenatal y el tipo de parto: una vía hacia el parto natural. Rev Chil Obstet Ginecol. 2006;71(2):98-103.

27. Smaill F, Hofmeyr GJ. Antibiotic prophylaxis for cesarean section. Cochrane Database Syst Rev. 2002(3):CD000933.

28. Ceriani-Cernadas J Mg, Pardo A, Aguirre A, Pérex C, Brener P, Cores F. Nacimiento por cesárea al término en embarazos de bajo riesgo: efectos sobre la morbilidad neonatal. Arch Argent Pediatr. 2010;108(1):17-23.

29. Tita AT, Landon MB, Spong CY, Lai Y, Leveno KJ, Varner MW, et al. Timing of elective repeat cesarean delivery at term and neonatal outcomes. The New England journal of medicine. $2009 ; 360(2): 111-20$

30. Gilbert WM, Nesbitt TS, Danielsen B. The cost of prematurity: quantification by gestational age and birth weight. Obstet Gynecol. 2003;102(3):488-92.

31. Tita AT, Lai Y, Landon MB, Spong CY, Leveno KJ, Varner MW, et al. Timing of elective repeat cesarean delivery at term and maternal perioperative outcomes. Obstet Gynecol. $2011 ; 117(2$ Pt 1$): 280-6$.

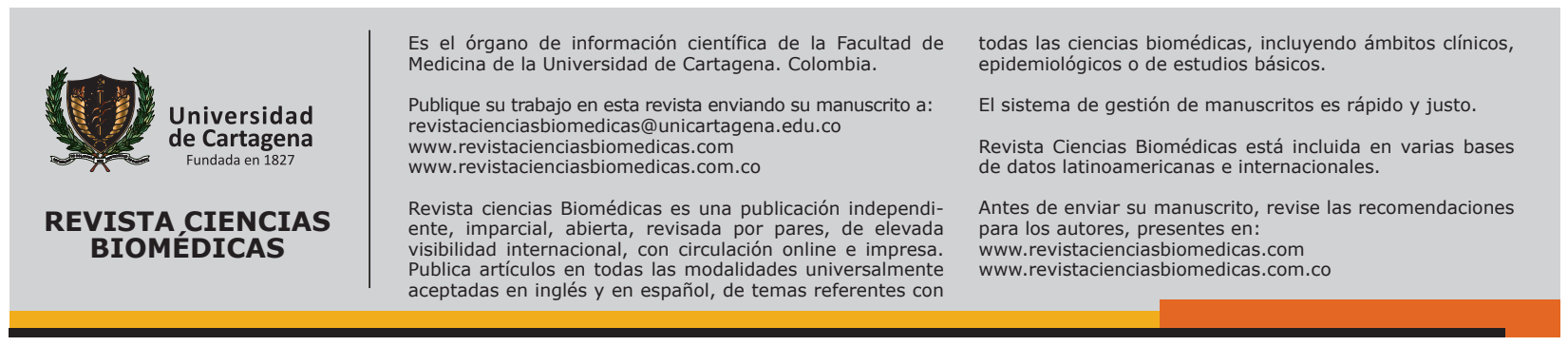

